Int. J. Electrochem. Sci., 14 (2019) $8836-8851$

\title{
The Novel biocatalytic cascade ZIF-8 capsule/Polysulfone Stereostructure and its Application in Amperometric Glucose Biosensors
}

\author{
Cang Wang ${ }^{1}$, Min Pan ${ }^{1}$, Hang Chen ${ }^{1}$, Dajing Chen ${ }^{2, *}$, Yuquan Chen ${ }^{1, *}$ \\ ${ }^{1}$ Department of Biomedical Engineering, Zhejiang University, Hangzhou 310027, China \\ ${ }^{2}$ Medical School, Hangzhou Normal University, Hangzhou, 311121, China \\ *E-mail: djchen@hznu.edu.cn
}

doi: $10.20964 / 2019.09 .13$

Received: 7 May 2019 / Accepted: 21 June 2019 / Published: 31 July 2019

\begin{abstract}
In this study, we present a novel zeolitic imidazolate framework-8 metal-organic framework /polysulfone (PSF) stereostructure with glucose oxidase (GOx) and horseradish peroxidase (HRP) encapsulated in ZIF-8 as nanoreactors. This work links the high biocatalytic activity and great stability of the enzymes encapsulated in ZIF-8 with the large surface area and mass transport of the threedimensional microporous PSF and highlights the pore structure of PSF, which blocks cells during whole blood testing. The prepared ZIF-8 capsule/PSF structure exhibits a 1.2-fold and 2.4-fold increase in sensitivity compared with the ZIF-8 capsule powder and bulk mixture of GOx and HRP, respectively. The modified ZIF-8 capsule/PSF glucose biosensor exhibited a 0-18 mM linear range and good selectivity. Furthermore, the prepared sensor attenuated less than $10 \%$ after 50 replicate whole blood tests. These advanced properties give the novel biocatalytic cascade ZIF-8 capsule/polysulfone stereostructure promising applications in whole blood monitoring.
\end{abstract}

Keywords: ZIF-8; nanostructure; glucose oxidase; horseradish peroxidase; glucose biosensor.

\section{FULL TEXT}

(C) 2019 The Authors. Published by ESG (www.electrochemsci.org). This article is an open access article distributed under the terms and conditions of the Creative Commons Attribution license (http://creativecommons.org/licenses/by/4.0/). 